CUBO A Mathematical Journal

Vol.20, $N^{\underline{O}_{3}}$, (13-29). October 2018

\title{
Mean curvature flow of certain kind of isoparametric foliations on non-compact symmetric spaces
}

\author{
NAOYUKI KoIKE \\ Department of Mathematics, Faculty of Science, \\ Tokyo University of Science, \\ 1-3 Kagurazaka Shinjuku-ku, Tokyo 162-8601, Japan \\ koike@rs.kagu.tus.ac.jp
}

\begin{abstract}
In this paper, we investigate the mean curvature flows starting from all leaves of the isoparametric foliation given by a certain kind of solvable group action on a symmetric space of non-compact type. We prove that the mean curvature flow starting from each non-minimal leaf of the foliation exists in infinite time, if the foliation admits no minimal leaf, then the flow asymptotes the self-similar flow starting from another leaf, and if the foliation admits a minimal leaf (in this case, it is shown that there exists the only one minimal leaf), then the flow converges to the minimal leaf of the foliation in $\mathrm{C}^{\infty}$-topology. These results give the geometric information between the leaves.
\end{abstract}

\section{RESUMEN}

En este artículo, investigamos el flujo por curvatura media comenzando desde cualquier hoja de una foliación isoparamétrica dada por la acción de un cierto grupo soluble en un espacio simétrico de tipo no-compacto. Demostramos que el flujo por curvatura media comenzando desde cualquier hoja no mínima de la foliación existe para tiempo infinito, si la foliación no admite hojas mínimas, entonces el flujo es asintótico al flujo autosemejante comenzando desde otra hoja; en cambio si el flujo admite una hoja mínima (en este caso, se muestra que la hoja mínima es única), entonces el flujo converge a dicha hoja mínima de la foliación en la topología $C^{\infty}$. Estos resultados entregan información geométrica entre las hojas.

Keywords and Phrases: error function based activation function, multivariate quasi-interpolation neural network approximation, Kantorovich-Shilkret type operator.

2010 AMS Mathematics Subject Classification: 41A17, 41A25, 41A30, 41A35. 


\section{Introduction}

In [6], we proved that the mean curvature flow starting from any non-minimal compact isoparametric (equivalently, equifocal) submanifold in a symmetric space of compact type collapses to one of its focal submanifolds in finite time. Here we note that parallel submanifolds and focal ones of the isoparametric submanifold give an isoparametric foliation consisting of compact leaves on the symmetric space, where an isoparametric foliation means a singular Riemannian foliation satisfying the following conditions:

(i) The mean curvature form is basic,

(ii) The regular leaves are submanifolds with section.

A singular Riemannian foliation satisfying only the first condition is called a generalized isoparametric foliation. Recently, M. M. Alexandrino and M. Radeschi [1] investigated the mean curvature flow starting from a regular leaf of a generalized isoparametric foliation consisting of compact leaves on a compact Riemannian manifold. In particular, they 1 generalized our result to the mean curvature flow starting from a regular leaf of the foliation in the case where the foliation is isoparametric and the ambient space curves non-negatively. On the other hand, we [7] proved that the mean curvature flow starting from a certain kind of non-minimal (not necessarily compact) isoparametric submanifold in a symmetric space of non-compact type (which curves non-positively) collapses to one of its focal submanifolds in finite time. Here we note that the isoparametric foliation associated with this isoparametric submanifold consists of curvature-adapted leaves. See the next paragraph about the definition of the curvature-adaptedness.

In this paper, we study the mean curvature flow starting from leaves of the isoparametiric foliation given by the action of a certain kind of solvable subgroup (see Examples 1 and 2) of the (full) isometry group of a symmetric space of non-compact type. Here we note that this isoparametric foliation consists of (not necessarily curvature-adapted) non-compact regular leaves. We shall explain the solvable group action which we treat in this paper. Let $\mathrm{G} / \mathrm{K}$ be a symmetric space of non-compact type, $\mathfrak{g}=\mathfrak{k}+\mathfrak{p}(\mathfrak{k}:=$ Lie $\mathrm{K})$ be the Cartan decomposition associated with the symmetric pair $(G, K), \mathfrak{a}$ be the maximal abelian subspace of $\mathfrak{p}, \widetilde{\mathfrak{a}}$ be the Cartan subalgebra of $\mathfrak{g}$ containing $\mathfrak{a}$ and $\mathfrak{g}=\mathfrak{k}+\mathfrak{a}+\mathfrak{n}$ be the Iwasawa's decomposition. Let $A, \widetilde{A}$ and $N$ be the connected Lie subgroups of $G$ having $\mathfrak{a}, \tilde{\mathfrak{a}}$ and $\mathfrak{n}$ as their Lie algebras, respectively. Let $\pi: G \rightarrow G / K$ be the natural projection.

Given metric. In this paper, we give G/K the G-invariant metric induced from the restriction $\left.B\right|_{\mathfrak{p} \times \mathfrak{p}}$ of the Killing form $B$ of $\mathfrak{g}$ to $\mathfrak{p} \times \mathfrak{p}$.

The symmetric space $G / K$ is identified with the solvable group $A N$ with a left-invariant metric through $\left.\pi\right|_{\text {AN }}$. Fix a lexicographic ordering of $\mathfrak{a}$. Let $\mathfrak{g}=\mathfrak{g}_{0}+\sum_{\lambda \in \Delta} \mathfrak{g}_{\lambda}, \mathfrak{p}=\mathfrak{a}+\sum_{\lambda \in \Delta_{+}} \mathfrak{p}_{\lambda}$ and 
$\mathfrak{k}=\mathfrak{k}_{0}+\sum_{\lambda \in \triangle_{+}} \mathfrak{k}_{\lambda}$ be the root space decompositions of $\mathfrak{g}, \mathfrak{p}$ and $\mathfrak{k}$ with respect to $\mathfrak{a}$, where we note that

$$
\begin{aligned}
& \mathfrak{g}_{\lambda}=\{X \in \mathfrak{g} \mid \operatorname{ad}(\mathfrak{a}) X=\lambda(\mathfrak{a}) X \text { for all } \mathfrak{a} \in \mathfrak{a}\} \quad(\lambda \in \triangle), \\
& \mathfrak{p}_{\lambda}=\left\{X \in \mathfrak{p} \mid \operatorname{ad}(\mathfrak{a})^{2} X=\lambda(a)^{2} X \text { for all } \mathfrak{a} \in \mathfrak{a}\right\} \quad\left(\lambda \in \triangle_{+}\right), \\
& \mathfrak{k}_{\lambda}=\left\{X \in \mathfrak{k} \mid \operatorname{ad}(a)^{2} X=\lambda(a)^{2} X \text { for all } \mathfrak{a} \in \mathfrak{a}\right\} \quad\left(\lambda \in \triangle_{+} \cup\{0\}\right) .
\end{aligned}
$$

Note that $\mathfrak{n}=\sum_{\lambda \in \triangle_{+}} \mathfrak{g}_{\lambda}$. Let $\mathrm{G}=\mathrm{KAN}$ be the Iwasawa decomposition of $\mathrm{G}$. Now we shall give examples of a solvable group contained in $A N$ whose action on $G / K(=A N)$ is (complex) hyperpolar. Since $G / K$ is of non-compact type, $\pi$ gives a diffeomorphism of $A N$ onto $G / K$. Denote by $\langle$,$\rangle the left-invariant metric of A N$ induced from the metric of $G / K$ by $\left.\pi\right|_{A N}$. Also, denote by $\langle,\rangle^{\mathrm{G}}$ the bi-invariant metric of $\mathrm{G}$ induced from the Killing form B. Note that $\langle,\rangle \neq \mathrm{\imath}^{*}\langle,\rangle^{\mathrm{G}}$, where $\iota$ is the inclusion map of $A N$ into $G$. Denote by Exp the exponential map of the Riemannian manifold $A N(=G / K)$ at $e$ and by $\exp _{G}$ the exponential map of the Lie group $G$. Let $l$ be a r-dimensional subspace of $\mathfrak{a}+\mathfrak{n}$ and set $\mathfrak{s}:=(\mathfrak{a}+\mathfrak{n}) \ominus l$, where $(\mathfrak{a}+\mathfrak{n}) \ominus l$ denotes the orthogonal complement of $l$ in $\mathfrak{a}+\mathfrak{n}$ with respect to $\langle,\rangle_{e}(e:$ is the identity element of $G)$. According to the result in [5], if $\mathfrak{s}$ is a subalgebra of $\mathfrak{a}+\mathfrak{n}$ and $l_{\mathfrak{p}}:=\operatorname{pr}_{\mathfrak{p}}(l)\left(\operatorname{pr}_{\mathfrak{p}}:\right.$ the orthogonal projection of $\mathfrak{g}$ onto $\mathfrak{p})$ is abelian, then the $\mathrm{S}$-action $\left(\mathrm{S}:=\exp _{\mathrm{G}}(\mathfrak{s})\right)$ gives an isoparametric foliation without singular leaf. We [5] gave examples of such a subalgebra $\mathfrak{s}$ of $\mathfrak{a}+\mathfrak{n}$.

Example 1. Let $\mathfrak{b}$ be a $r(\geq 1)$-dimensional subspace of $\mathfrak{a}$ and $\mathfrak{s}_{\mathfrak{b}}:=(\mathfrak{a}+\mathfrak{n}) \ominus \mathfrak{b}$. It is clear that $\mathfrak{b}_{\mathfrak{p}}(=\mathfrak{b})$ is abelian and that $\mathfrak{s}_{\mathfrak{b}}$ is a subalgebra of $\mathfrak{a}+\mathfrak{n}$.

Example 2. Let $\left\{\lambda_{1}, \cdots, \lambda_{k}\right\}$ be a subset of a simple root system $\Pi$ of $\triangle$ such that $H_{\lambda_{1}}, \cdots, H_{\lambda_{k}}$ are mutually orthogonal, $\mathfrak{b}$ be a subspace of $\mathfrak{a} \ominus \operatorname{Span}\left\{\mathrm{H}_{\lambda_{1}}, \cdots, \mathrm{H}_{\lambda_{k}}\right\}$ (where $\mathfrak{b}$ may be $\{0\}$ ) and $l_{i}(i=1, \cdots, k)$ be a one-dimensional subspace of $\mathrm{RH}_{\lambda_{i}}+\mathfrak{g}_{\lambda_{i}}$ with $l_{i} \neq R H_{\lambda_{i}}$, where $\mathrm{H}_{\lambda_{i}}$ is the element of $\mathfrak{a}$ defined by $\left\langle\mathrm{H}_{\lambda_{i}}, \cdot\right\rangle=\lambda_{\mathfrak{i}}(\cdot)$ and $\mathrm{RH}_{\lambda_{i}}$ is the subspace of $\mathfrak{a}$ spanned by $\mathrm{H}_{\lambda_{i}}$. Set $l:=\mathfrak{b}+\sum_{\mathfrak{i}=1}^{\mathrm{k}} l_{\mathfrak{i}}$. Then, it is shown that $l_{\mathfrak{p}}$ is abelian and that $\mathfrak{s}_{\mathfrak{b}, l_{1}, \cdots, l_{\mathfrak{k}}}:=(\mathfrak{a}+\mathfrak{n}) \ominus l$ is a subalgebra of $\mathfrak{a}+\mathfrak{n}$.

In Example 2, a unit vector of $l_{i}$ is described as $\frac{1}{\cosh \left(\left\|\lambda_{i}\right\| t_{i}\right)} \xi^{i}-\frac{1}{\left\|\lambda_{i}\right\|} \tanh \left(\left\|\lambda_{i}\right\| t_{i}\right) H_{\lambda_{i}}$ for a unit vector $\xi^{i}$ of $\mathfrak{g}_{\lambda_{i}}$ and some $t_{i} \in \mathbb{R}$, where $\left\|\lambda_{i}\right\|:=\left\|\mathrm{H}_{\lambda_{i}}\right\|$. Then we denote $l_{i}$ by $l_{\xi^{i}, \mathfrak{t}_{i}}$ if necessary and set $\xi_{\mathfrak{t}_{i}}^{i}:=\frac{1}{\cosh \left(\left\|\lambda_{i}\right\| t_{i}\right)} \xi^{i}-\frac{1}{\left\|\lambda_{i}\right\|} \tanh \left(\left\|\lambda_{i}\right\| t_{\mathfrak{i}}\right) H_{\lambda_{i}}$. Set $S_{\mathfrak{b}}:=\exp _{G}\left(\mathfrak{s}_{\mathfrak{b}}\right)$ and $S_{\mathfrak{b}, l_{1}, \cdots, l_{k}}:=$ $\exp _{\mathrm{G}}\left(\mathfrak{s}_{\mathfrak{b}, l_{1}, \cdots, l_{k}}\right)$. Denote by $\mathfrak{F}_{\mathfrak{b}}$ and $\mathfrak{F}_{\mathfrak{b}, l_{1}, \cdots, l_{k}}$ the isoparametric foliations given by the $\boldsymbol{S}_{\mathfrak{b}}$-action and the $S_{\mathfrak{b}, l_{1}, \cdots, l_{k}}$-one, respectively. A submanifold in a Riemannian manifold is said to be curvature-adapted if, for each normal vector $v$ of the submanifold, the normal Jacobi operator $\mathrm{R}(v):=\mathrm{R}(\cdot, v) v$ preserves the tangent space of the submanifold invariantly and the restriction of $\mathrm{R}(v)$ to the tangent space commutes with the shape operator $\mathrm{A}_{v}$, where $\mathrm{R}$ is the curvature tensor of the ambient Riemannian manifold. According to the results in $[5]$, the following facts hold for 
isoparametric foliations $\mathfrak{F}_{\mathfrak{b}}$ and $\mathfrak{F}_{\mathfrak{b}, l_{1}, \cdots, l_{k}}$ :

(i) All leaves of $\mathfrak{F}_{\mathfrak{b}}$ are curvature-adapted.

(ii) Let $\lambda_{1}, \cdots, \lambda_{k}\left(\in \triangle_{+}\right)$be as in Example 2. If the root system $\triangle$ of $G / K$ is non-reduced and $2 \lambda_{i_{0}} \in \triangle_{+}$for some $i_{0} \in\{1, \cdots, k\}$, then all leaves of $\mathfrak{F}_{\mathfrak{b}, l_{1}, \cdots, l_{k}}$ are not curvature-adapted.

(iii) If $\mathfrak{b} \neq\{0\}$, then $\mathfrak{F}_{\mathfrak{b}, l_{1}, \cdots, l_{k}}$ admits no minimal leaf. On the other hand, if $\mathfrak{b}=\{0\}$, then this action admits the only minimal leaf.

(iv) Let $l_{1}, \cdots, l_{k}$ be as in Example 2 and $\bar{l}_{i}(i=1, \cdots, k)$ be the orthogonal projection of $l_{i}$ onto $\mathfrak{g}_{\lambda_{i}}$. Then $\mathfrak{F}_{\mathfrak{b}, \bar{l}_{1}, \ldots, \bar{l}_{\mathfrak{k}}}$ is congruent to $\mathfrak{F}_{\mathfrak{b}, l_{1}, \ldots, l_{k}}$. In more detail, we have

$$
\mathrm{L}_{\mathrm{b} \cdot \gamma_{\xi_{1}}\left(\mathrm{t}_{1}\right) \cdots \cdot \gamma_{\xi^{k}}\left(\mathrm{t}_{\mathrm{k}}\right)}\left(\mathrm{S}_{\mathfrak{b}, l_{1}, \cdots, l_{k}} \cdot e\right)=\mathrm{S}_{\mathfrak{b}, \bar{l}_{1}, \cdots, \bar{l}_{k}} \cdot\left(\mathrm{b} \cdot \gamma_{\xi^{1}}\left(\mathrm{t}_{1}\right) \cdots \cdot \gamma_{\xi^{k}}\left(\mathrm{t}_{\mathrm{k}}\right)\right) \text {, }
$$

where $\gamma_{\xi^{i}}(i=1, \cdots, k)$ is the geodesic in $A N(=G / K)$ with $\gamma_{\xi^{i}}^{\prime}(0)=\xi^{i}, b$ is an element of $\exp (\mathfrak{b})$ and $L_{b \cdot \gamma_{\xi_{1}}}\left(t_{1}\right) \cdots \cdot \gamma_{\xi^{k}}\left(t_{k}\right)$ is the left translation by $b \cdot \gamma_{\xi^{1}}\left(t_{1}\right) \cdots \cdot \gamma_{\xi^{k}}\left(t_{k}\right)$. For example, in case of $k=1$ and $b=e$, the positional relation among the leaves of these foliations is as in Figure 1.

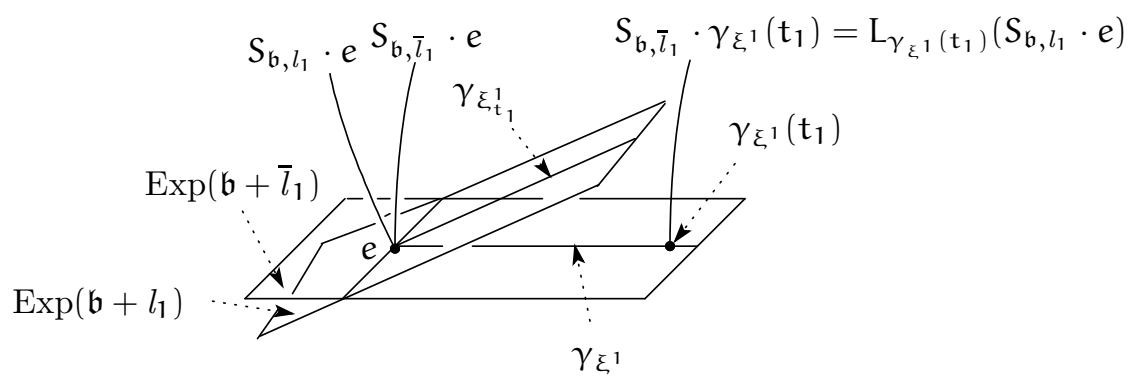

Figure 1.

According to the above facts (i) and (ii), the leaves of $\mathfrak{F}_{\mathfrak{b}, l_{1}, \cdots, l_{k}}$ give examples of interesting isoparametric submanifolds in $\mathrm{G} / \mathrm{K}$.

In this paper, we shall prove the following facts for the mean curvature flows starting from the non-minimal leaves of $\mathfrak{F}_{\mathfrak{b}, \bar{l}_{1}, \cdots, \bar{l}_{k}}$.

Theorem A. Assume that $\mathfrak{b} \neq\{0\}$. Let $M$ be any leaf of $\mathfrak{F}_{\mathfrak{b}, \bar{l}_{1}, \ldots, \bar{l}_{k}}$. and $\mathrm{M}_{\mathrm{t}}(0 \leq \mathrm{t}<\mathrm{T})$ be the mean curvature flow starting from $M$. Then the following statements (i) - (iii) hold.

(i) $\mathrm{T}=\infty$ holds.

(ii) If $M$ passes through $\exp (\mathfrak{b})$, then the mean curvature flow $M_{t}$ is self-similar.

(iii) If $M$ does not pass through $\exp (\mathfrak{b})$, then the mean curvature flow $M_{t}$ asymptotes the mean curvature flow starting from the leaf of $\mathfrak{F}_{\mathfrak{b}, \bar{l}_{1}, \ldots, \bar{l}_{k}}$ passing through a point of $\exp (\mathfrak{b})$.

Remark 1.1. The mean curvature flow starting from any leaf of $\mathfrak{F}_{\mathfrak{b}}$ is self-similar. 


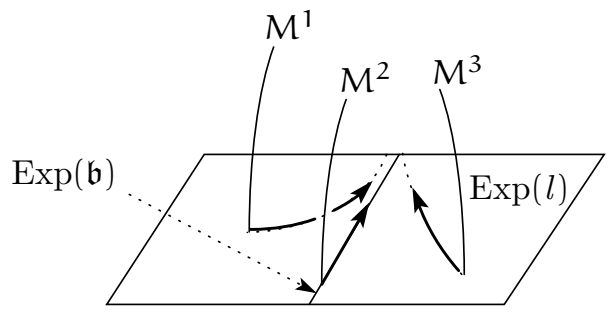

The mean curvature flows starting from leaves $M^{1}$ and $M^{3}$

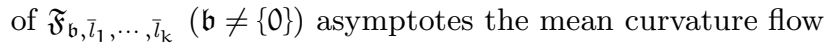
(which is self-similar) starting from a leaf $\mathrm{M}^{2}$ of $\mathfrak{F}_{\mathfrak{b}, \bar{l}_{1}, \ldots, \bar{l}_{\mathrm{k}}}$.

Figure 2 .

Also, in case of $\mathfrak{b}=\{0\}$, we obtain the following fact.

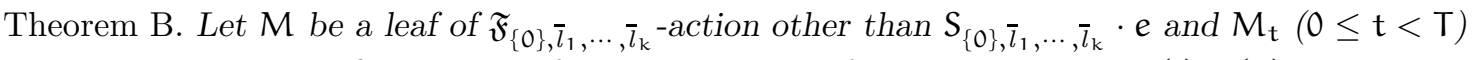
be the mean curvature flow starting from $M$. Then the following statements (i) - (ii) hold.

(i) $\mathrm{T}=\infty$ holds.

(ii) $\mathrm{M}_{\mathrm{t}}$ convergres to the only minimal leaf $\mathrm{S}_{\{0\}, \bar{l}_{1}, \ldots, \bar{l}_{\mathrm{k}}} \cdot \mathrm{e}$ (in $\mathrm{C}^{\infty}$-topology) as $\mathrm{t} \rightarrow \infty$.

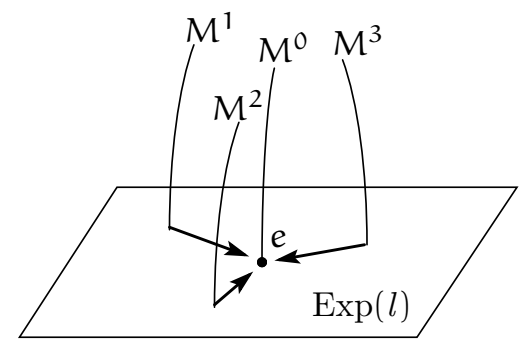

The mean curvature flows starting from leaves $M^{1}, M^{2}$ and $M^{3}$ of $\mathfrak{F}_{\{0\}, \bar{l}_{1}, \cdots, \bar{l}_{\mathrm{k}}}$ converge to the only minimal leaf $\mathrm{M}^{0}$ of $\mathfrak{F}_{\{0\}, \bar{l}_{1}, \cdots, \bar{l}_{\mathrm{k}}}$.

Figure 3.

The following question arises naturally.

Question. Let $\mathfrak{F}$ be an isoparametric foliation consisting of non-compact regular leaves on a nonpositively curved Riemannian manifold. Assume that the leaves of $\mathfrak{F}$ are cohomogeneity compact (i.e., each leaf $\mathrm{L}$ is invariant under some subgroup action $\mathrm{H}_{\mathrm{L}}$ of the isometry group of the ambient space and the quotient space $\mathrm{L} / \mathrm{H}_{\mathrm{L}}$ is compact). In what case, does the result similar to Theorem $A$ or $B$ hold for $\mathfrak{F}$ ? 


\section{Mean curvature flow.}

In this section, we shall recall the notion of the mean curvature flow. Let $f_{t}$ 's $(t \in[0, T))$ be a oneparameter $C^{\infty}$-family of immersions of a manifold $M$ into a Riemannian manifold $\widetilde{M}$, where $T$ is a positive constant or $T=\infty$. Define a map $F: M \times[0, T) \rightarrow \widetilde{M}$ by $F(x, t)=f_{t}(x)((x, t) \in M \times[0, T))$. Denote by $\pi$ the natural projection of $M \times[0, T)$ onto $M$. For a vector bundle $E$ over $M$, denote by $\pi^{*} E$ the induced bundle of $E$ by $\pi$. Also, denote by $H_{t}$ and $g_{t}$ the mean curvature vector field and the induced metric of $f_{t}$, respectively. Define a section $g$ of $\pi^{*}\left(T^{(0,2)} M\right)$ by $g_{(x, t)}:=\left(g_{t}\right)_{x}$ $((x, t) \in M \times[0, T))$ and sections $H$ of $F^{*} T \widetilde{M}$ by $H_{(x, t)}:=\left(H_{t}\right)_{x}((x, t) \in M \times[0, T))$, where $T^{(0,2)} M$ is the tensor bundle of degree $(0,2)$ of $M$ and $T \widetilde{M}$ is the tangent bundle of $\widetilde{M}$. The family $f_{t}$ 's $(0 \leq \mathrm{t}<\mathrm{T})$ is called a mean curvature flow if it satisfies

$$
F_{*}\left(\frac{\partial}{\partial t}\right)=H .
$$

In particular, if $f_{t}$ 's are embeddings, then we call $M_{t}:=f_{t}(M)$ 's $(0 \in[0, T))$ rather than $f_{t}$ 's $(0 \leq \mathrm{t}<\mathrm{T})$ a mean curvature flow. See [3, [4] and [2] and so on about the study of the mean curvature flow (treated as the evolution of an immersion).

\section{The non-curvature-adaptedness of the leaves.}

In [5], we proved the following statement:

(*) If the root system $\triangle$ of $\mathrm{G} / \mathrm{K}$ is non-reduced and $2 \lambda_{i_{0}} \in \triangle_{+}$for some $i_{0} \in\{1, \cdots, k\}$, then all leaves of $\mathfrak{F}_{\mathfrak{b}, \bar{l}_{1}, \ldots, \bar{l}_{\mathfrak{k}}}$ are not curvature-adapted.

(see the statement (ii) of Proposition 3.5 in [5]). However, there is a gap in the second-half part of the proof. In this section, we shall close the gap by recalculating the normal Jacobi operators of the leaves (see Proposition 3.5). We shall use the notations in Introduction. According to the fact (iv) stated in Introduction, we have

$$
\mathrm{L}_{\mathrm{b} \cdot \gamma_{\xi_{1}}\left(\mathrm{t}_{1}\right) \cdots \cdot \gamma_{\xi^{k}}\left(\mathrm{t}_{\mathrm{k}}\right)}\left(\mathrm{S}_{\mathfrak{b}, l_{1}, \cdots, l_{k}} \cdot e\right)=\mathrm{S}_{\mathfrak{b}, \bar{l}_{1}, \ldots, \bar{l}_{k}} \cdot\left(\mathrm{b} \cdot \gamma_{\xi^{1}}\left(\mathrm{t}_{1}\right) \cdots \cdot \gamma_{\xi^{k}}\left(\mathrm{t}_{\mathrm{k}}\right)\right) \text {. }
$$

Hence we suffice to show that the leaves $S_{\mathfrak{b}, l_{1}, \ldots, l_{k}} \cdot e^{\prime}$ s are not curvature-adapted. As stated in Example 2, we set $\xi_{t_{i}}^{i}:=\frac{1}{\cosh \left(\left\|\lambda_{i}\right\| t_{i}\right)} \xi^{i}-\frac{1}{\left\|\lambda_{i}\right\|} \tanh \left(\left\|\lambda_{i}\right\| t_{i}\right) H_{\lambda_{i}}$. For the shape operator of $S_{\mathfrak{b}, l_{1}, \cdots, l_{k}} \cdot e$, we showed the following facts (see Lemma 3.2 of [5]).

Lemma 3.1[5]. Let $A$ be the shape tensor of $S_{\mathfrak{b}, l_{1}, \cdots, l_{k}} \cdot e(\subset A N)$. Then, for $A_{\xi_{0}}\left(\xi_{0} \in \mathfrak{b}\right)$ and $A_{\xi_{t_{i}}^{i}}(i=1, \cdots, k)$, the following statements (i) (vii) hold:

(i) For $X \in \mathfrak{a} \ominus\left(\mathfrak{b}+\sum_{i=1}^{k} R H_{\lambda_{i}}\right)$, we have $A_{\xi_{0}} X=A_{\xi_{\mathfrak{t}_{i}}^{i}} X=0(i=1, \cdots, k)$. 
(ii) For $\mathrm{X} \in \operatorname{Ker}\left(\left.\operatorname{ad}\left(\xi^{i}\right)\right|_{\mathfrak{g}_{\lambda_{i}}}\right) \ominus R \xi^{i}$, we have $A_{\xi_{0}} X=0$ and $A_{\xi_{t_{i}}^{i}} X=-\left\|\lambda_{i}\right\| \tanh \left(\left\|\lambda_{i}\right\| t_{i}\right) X$.

(iii) Assume that $2 \lambda_{i} \in \triangle_{+}$. For $X \in \mathfrak{g}_{2 \lambda_{i}}$, we have $A_{\xi_{0}}\left(\left[\theta \xi^{i}, X\right]\right)=0$ and

$$
\begin{aligned}
& A_{\xi_{t_{i}}^{i}} X=-2\left\|\lambda_{i}\right\| \tanh \left(\left\|\lambda_{i}\right\| t_{i}\right) X-\frac{1}{2 \cosh \left(\left\|\lambda_{i}\right\| t_{i}\right)}\left[\theta \xi^{i}, X\right], \\
& A_{\xi_{t_{i}}^{i}}\left(\left[\theta \xi^{i}, X\right]\right)=-\frac{\left\|\lambda_{i}\right\|^{2}}{\cosh \left(\left\|\lambda_{i}\right\| t_{i}\right)} X-\left\|\lambda_{i}\right\| \tanh \left(\left\|\lambda_{i}\right\| t_{i}\right)\left[\theta \xi^{i}, X\right],
\end{aligned}
$$

where $\theta$ is the Cartan involution of $\mathfrak{g}$ with Fix $\theta=\mathfrak{k}$.

(iv) For $\mathrm{X} \in\left(R \xi^{i}+R H_{\lambda_{i}}\right) \ominus l_{i}$, we have $A_{\xi_{0}} X=0$ and $A_{\xi_{t_{i}}} X=-\left\|\lambda_{i}\right\| \tanh \left(\left\|\lambda_{i}\right\| t_{i}\right) X$.

(v) For $X \in\left(\mathfrak{g}_{\lambda_{j}} \ominus R \xi^{j}\right)+\left(\left(R \xi^{j}+R H_{\lambda_{j}}\right) \ominus l_{j}\right)+\mathfrak{g}_{2 \lambda_{j}}(j \neq i)$, we have $A_{\xi_{0}} X=A_{\xi_{t_{i}}^{i}} X=0$.

(vi) For $X \in \mathfrak{g}_{\mu}\left(\mu \in \triangle_{+} \backslash\left\{\lambda_{1}, \cdots, \lambda_{k}\right\}\right)$, we have $A_{\xi_{0}} X=\mu\left(\xi_{0}\right) X$.

(vii) Let $k_{i}:=\exp \left(\frac{\pi}{\sqrt{2}\left\|\lambda_{i}\right\|}\left(\xi^{i}+\theta \xi^{i}\right)\right)$, where exp is the exponential map of G. Then $\operatorname{Ad}\left(k_{i}\right) \circ$ $A_{\xi_{t_{i}}^{i}}=-A_{\xi_{t_{i}}^{i}} \circ \operatorname{Ad}\left(k_{i}\right)$ holds over $\mathfrak{n} \ominus \sum_{i=1}^{k}\left(\mathfrak{g}_{\lambda_{i}}+\mathfrak{g}_{2 \lambda_{i}}\right)$, where Ad is the adjoint representation of $\mathrm{G}$.

Remark 3.1. If $\lambda_{i} \in \triangle_{+}$, then we have $\left\|\lambda_{i}\right\|=\sqrt{2}$ from how to choose the metric of $G / K$ (see Introduction).

According to (5.3) in Page 310 of [8], we have the following fact.

Lemma $3.2[8]$. Let $\mathrm{X}$ and $\mathrm{Y}$ be left-invariant vector fields on $\mathrm{AN}$ and $\nabla$ be the Levi-Civita connection of the left-invariant metric $\langle$,$\rangle of \mathrm{AN}$. Then we have

$$
\nabla_{X} Y=\frac{1}{2}\left([X, Y]-\operatorname{ad}(X)^{*}(Y)-\operatorname{ad}(Y)^{*}(X)\right)
$$

where $\operatorname{ad}(\mathrm{X})^{*}\left(\operatorname{resp} \cdot \operatorname{ad}(\mathrm{Y})^{*}\right)$ is the adjoint operator of $\operatorname{ad}(\mathrm{X})(\operatorname{resp} \cdot \operatorname{ad}(\mathrm{Y}))$ with respect to $\langle,\rangle_{e}$ and $(\bullet)_{\mathfrak{a}+\mathfrak{n}}$ is the the $(\mathfrak{a}+\mathfrak{n})$-component of $(\bullet)$.

Let $\operatorname{pr}_{\mathfrak{a}+\mathfrak{n}}^{1}\left(\right.$ resp. $\left.\operatorname{pr}_{\mathfrak{a}+\mathfrak{n}}^{2}\right)$ be the projection of $\mathfrak{g}$ onto $\mathfrak{a}+\mathfrak{n}$ with respect to the decomposition $\mathfrak{g}=\mathfrak{k}+(\mathfrak{a}+\mathfrak{n})\left(\right.$ resp. $\left.\mathfrak{g}=\left(\mathfrak{k}_{0}+\sum_{\lambda \in \triangle_{+}} \mathfrak{p}_{\lambda}\right)+(\mathfrak{a}+\mathfrak{n})\right)$. We [5] showed the following facts (see the proof of Lemma 3.2 in [5]).

Lemma 3.3[5]. (i) For any $\mathrm{H} \in \mathfrak{a}$, we have

$$
\operatorname{ad}(H)^{*}=\operatorname{ad}(H) .
$$


(ii) For any $X \in \mathfrak{g}_{\lambda}$, we have

$$
\begin{aligned}
\operatorname{ad}(X)^{*} & =-\operatorname{pr}_{\mathfrak{a}+\mathfrak{n}} \circ \operatorname{ad}(\theta X) \\
& = \begin{cases}0 & \text { on } \mathfrak{a} \\
-\langle X,\rangle_{\mathfrak{e}} \otimes \mathrm{H}_{\lambda}-\operatorname{pr}_{\mathfrak{n}} \circ \operatorname{pr}_{\mathfrak{a}+\mathfrak{n}}^{1} \circ \operatorname{ad}\left(X_{\mathfrak{k}}\right) & \text { on } \mathfrak{n}, \\
+\operatorname{pr}_{\mathfrak{n}} \circ \operatorname{pr}_{\mathfrak{a}+\mathfrak{n}}^{2} \circ \operatorname{ad}\left(X_{\mathfrak{p}}\right) & \end{cases}
\end{aligned}
$$

where $(\bullet)_{\mathfrak{k}}\left(\right.$ resp. $\left.(\cdot)_{\mathfrak{p}}\right)$ denotes the $\mathfrak{k}$-component (resp. p-component) of $(\bullet)$.

According to (3.4), we have

$$
\operatorname{ad}(X)^{*}(Y)= \begin{cases}0 & \left(\lambda-\mu \in \triangle_{+}\right) \\ -\langle X, Y\rangle H_{\lambda} & (\lambda=\mu) \\ -[\theta X, Y] & \left(\mu-\lambda \in \triangle_{+}\right) \\ 0 & (\lambda-\mu \notin \triangle \cup\{0\})\end{cases}
$$

for any $X \in \mathfrak{g}_{\lambda}\left(\lambda \in \triangle_{+}\right)$and any $Y \in \mathfrak{g}_{\mu}\left(\mu \in \triangle_{+}\right)$. For each $X \in \mathfrak{a}+\mathfrak{n}$, we denote by $\widetilde{X}$ the left-invariant vector field on $A N$ with $(\tilde{X})_{e}=X$. By using Lemma 3.2, (3.3), (3.4) and (3.5), we can derive the facts directly.

Lemma 3.4. For any unit vector $X_{\lambda}, Y_{\lambda}$ of $\mathfrak{g}_{\lambda}\left(\lambda \in \triangle_{+}\right)$and $\mathrm{H}_{\lambda}\left(\lambda \in \triangle_{+}\right)$, we have

$$
\nabla_{\widetilde{H}_{\lambda}} \widetilde{H}_{\mu}=\nabla_{\widetilde{H}_{\lambda}} \widetilde{X}_{\mu}=0, \quad \nabla_{\widetilde{X}_{\lambda}} \widetilde{H}_{\mu}=-\lambda\left(H_{\mu}\right) \widetilde{X}_{\lambda} \quad\left(\lambda, \mu \in \triangle_{+}\right)
$$

and

$$
\nabla_{\widetilde{X}_{\lambda}} \widetilde{Y}_{\mu}= \begin{cases}\frac{1}{2}\left(\left[\widetilde{X}_{\lambda}, \widetilde{Y}_{\mu}\right]+\widetilde{\theta\left[Y_{\mu}, \theta X_{\lambda}\right]}\right) & \left(\lambda-\mu \in \triangle_{+}\right) \\ \frac{1}{2}\left[\widetilde{X}_{\lambda}, \widetilde{Y}_{\mu}\right]+\left\langle\widetilde{X}_{\lambda}, \widetilde{Y}_{\mu}\right\rangle \widetilde{H}_{\lambda} & (\lambda=\mu) \\ \frac{1}{2}\left(\left[\widetilde{X}_{\lambda}, \widetilde{Y}_{\mu}\right]+\widetilde{\left.\theta\left[X_{\lambda}, \theta Y_{\mu}\right]\right)}\right. & \left(\mu-\lambda \in \triangle_{+}\right) \\ \frac{1}{2}\left[\widetilde{X}_{\lambda}, \widetilde{Y}_{\mu}\right] & (\lambda-\mu \notin \Delta \cup\{0\})\end{cases}
$$

From Lemma 3.4 and (3.5), we can derive the following facts for the normal Jacobi operators by somewhat long calculations.

Proposition 3.5. Let $R$ be the curvature tensor of $A N(=G / K)$. Then, for $R\left(\xi_{0}\right)\left(\xi^{0} \in \mathfrak{b}\right)$ and $R\left(\xi_{t_{i}}^{i}\right)(i=1, \cdots, k)$, the following statements (i) (vi) hold:

(i) For $X \in \mathfrak{a} \ominus\left(\mathfrak{b}+\sum_{i=1}^{k} R H_{\lambda_{i}}\right)$, we have $R\left(\xi_{0}\right)(X)=R\left(\xi_{\mathfrak{t}_{i}}^{i}\right)(X)=0(i=1, \cdots, k)$. 
(ii) For $X \in \operatorname{Ker}\left(\left.\operatorname{ad}\left(\xi^{i}\right)\right|_{\mathfrak{g}_{\lambda_{i}}}\right) \ominus R \xi^{i}$, we have $\mathrm{R}\left(\xi_{0}\right)(X)=0$ and $\mathrm{R}\left(\xi_{\mathfrak{t}_{i}}^{i}\right)(X)=\frac{\left\|\lambda_{i}\right\|^{2}}{2}(1-$ $\left.3 \tanh ^{2}\left(\left\|\lambda_{i}\right\| t_{i}\right)\right) X$.

(iii) Assume that $2 \lambda_{i} \in \triangle_{+}$(hence $\left\|\lambda_{i}\right\|=\sqrt{2}$ ). For $X \in \mathfrak{g}_{2 \lambda_{i}}$, we have $R\left(\xi_{0}\right)(X)=$ $\mathrm{R}\left(\xi_{0}\right)\left(\left[\theta \xi^{i}, X\right]\right)=0$ and

$$
\begin{aligned}
& R\left(\xi_{t_{i}}^{i}\right)(X)=-\left\|\lambda_{i}\right\|^{2}\left(1+3 \tanh ^{2}\left(\left\|\lambda_{i}\right\| t_{i}\right)\right) X-\frac{3\left\|\lambda_{i}\right\| \tanh \left(\left\|\lambda_{i}\right\| t_{i}\right)}{2 \cosh \left(\left\|\lambda_{i}\right\| t_{i}\right)}\left[\theta \xi^{i}, X\right] \\
& R\left(\xi_{t_{i}}^{i}\right)\left(\left[\theta \xi^{i}, X\right]\right)=-\frac{6\left\|\lambda_{i}\right\| \tanh \left(\left\|\lambda_{i}\right\| t_{i}\right)}{\cosh \left(\left\|\lambda_{i}\right\| t_{i}\right)} X+\frac{\sqrt{2}\left\|\lambda_{i}\right\|}{4}\left(1-3 \tanh ^{2}\left(\left\|\lambda_{i}\right\| t_{i}\right)\right)\left[\theta \xi^{i}, X\right] .
\end{aligned}
$$

(iv) For $\mathrm{X} \in\left(\mathrm{R} \xi^{i}+\mathrm{RH}_{\lambda_{i}}\right) \ominus l_{i}$, we have $\mathrm{R}\left(\xi_{0}\right)(\mathrm{X})=0$ and $\mathrm{R}\left(\xi_{\mathrm{t}_{i}}^{i}\right)(\mathrm{X})=-\left\|\lambda_{i}\right\|^{2} \mathrm{X}$.

(v) For $X \in\left(\mathfrak{g}_{\lambda_{j}} \ominus R \xi^{j}\right)+\left(\left(R \xi^{j}+R H_{\lambda_{j}}\right) \ominus l_{j}\right)+\mathfrak{g}_{2 \lambda_{j}}(j \neq i)$, we have $R\left(\xi_{0}\right)(X)=R\left(\xi_{\mathfrak{t}_{i}}^{i}\right)(X)=0$.

(vi) For $X \in \mathfrak{g}_{\mu}\left(\mu \in \triangle_{+} \backslash\left\{\lambda_{1}, \cdots, \lambda_{k}\right\}\right)$, we have $R\left(\xi_{0}\right)(X)=-\mu\left(\xi_{0}\right)^{2} X$.

From Lemma 3.1 and Proposition 3.5, we can derive the following facts directly.

Proposition 3.6. For $\left[A_{\xi_{0}}, R\left(\xi_{0}\right)\right]\left(\xi_{0} \in \mathfrak{b}\right)$ and $\left[A_{\xi_{t_{i}}^{i}}, R\left(\xi_{\mathfrak{t}_{i}}^{i}\right)\right](i=1, \cdots, k)$, the following statements (i) (vi) hold:

(i) For $X \in \mathfrak{a} \ominus\left(\mathfrak{b}+\sum_{i=1}^{k} R H_{\lambda_{i}}\right)$, we have $\left[A, R\left(\xi_{0}\right)\right](X)=\left[A_{\xi_{t_{i}}^{i}}, R\left(\xi_{\mathfrak{t}_{i}}^{i}\right)\right](X)=0(i=1, \cdots, k)$.

(ii) For $\mathrm{X} \in \operatorname{Ker}\left(\left.\operatorname{ad}\left(\xi^{i}\right)\right|_{\mathfrak{g}_{\lambda_{i}}}\right) \ominus R \xi^{i}$, we have $\left[A_{\xi_{0}}, R\left(\xi_{0}\right)\right](X)=\left[A_{\xi_{t_{i}}^{i}}, R\left(\xi_{t_{i}}^{i}\right)\right](X)=0$.

(iii) Assume that $2 \lambda_{i} \in \triangle_{+}$(hence $\left.\left\|\lambda_{i}\right\|=\sqrt{2}\right)$. For $X \in \mathfrak{g}_{2 \lambda_{i}}$, we have $\left[A_{\xi_{0}}, R\left(\xi_{0}\right)\right](X)=$ $\left[A_{\xi_{0}}, R\left(\xi_{0}\right)\right]\left(\left[\theta \xi^{i}, X\right]\right)=0$ and

$$
\begin{aligned}
& {\left[A_{\xi_{t_{i}}^{i}}, R\left(\xi_{t_{i}}^{i}\right)\right](X)=-\frac{3}{2 \cosh ^{3}\left(\sqrt{2} t_{i}\right)}\left[\theta \xi^{i}, X\right]} \\
& {\left[A_{\xi_{t_{i}}^{i}}, R\left(\xi_{t_{i}}^{i}\right)\right]\left(\left[\theta \xi^{i}, X\right]\right)=-\frac{6}{\cosh ^{3}\left(\sqrt{2} t_{i}\right)} X .}
\end{aligned}
$$

(iv) For $X \in\left(R \xi^{i}+R H_{\lambda_{i}}\right) \ominus l_{i}$, we have $\left[A_{\xi_{0}}, R\left(\xi_{0}\right)\right](X)=\left[A_{\xi_{t_{i}}^{i}}, R\left(\xi_{t_{i}}^{i}\right)\right](X)=0$.

(v) For $X \in\left(\mathfrak{g}_{\lambda_{j}} \ominus R \xi^{j}\right)+\left(\left(R \xi^{j}+R H_{\lambda_{j}}\right) \ominus l_{j}\right)+\mathfrak{g}_{2 \lambda_{j}}(j \neq i)$, we have $\left[A_{\xi_{0}}, R\left(\xi_{0}\right)\right](X)=$ $\left[A_{\xi_{t_{i}}^{i}}, R\left(\xi_{t_{i}}^{i}\right)\right](X)=0$.

(vi) For $X \in \mathfrak{g}_{\mu}\left(\mu \in \triangle_{+} \backslash\left\{\lambda_{1}, \cdots, \lambda_{k}\right\}\right)$, we have $\left[A_{\xi_{0}}, R\left(\xi_{0}\right)\right](X)=\left[A_{\xi_{t_{i}}^{i}}, R\left(\xi_{t_{i}}^{i}\right)\right](X)=0$.

From (iv) of Proposition 3.6, we can derive the statement $(*)$.

Also, we [5] showed the following fact in terms of Lemma 3.1. 
Proposition $3.7[5]$. If $\mathfrak{b}=\{0\}$, then $\mathfrak{F}_{\mathfrak{b}, l_{\xi^{1}, \mathrm{t}_{1}}, \cdots, l_{\xi^{k}, t_{k}}}$ admits the only minimal leaf.

\section{Proof of Theorem A}

In this section, we shall prove Theorem A. We use the notations in Sections 1 and 3. Note that $\left.\operatorname{Exp}\right|_{\mathfrak{a}}=\left.\exp \right|_{\mathfrak{a}}$ and $\left.\operatorname{Exp}\right|_{\mathfrak{n}} \neq\left.\exp \right|_{\mathfrak{n}}$. Set $\Sigma:=\operatorname{Exp}\left(\mathrm{T}_{e}^{\perp} S_{\mathfrak{b}, \bar{l}_{1}, \cdots, \bar{l}_{\mathfrak{k}}} \cdot e\right)\left(=\operatorname{Exp}\left(\mathfrak{b}+\mathbb{R}\left\{\xi^{1}, \cdots, \xi^{k}\right\}\right)\right)$, which is the flat section of the $S_{\mathfrak{b}, \bar{l}_{1}, \cdots, \bar{l}_{\mathfrak{k}}}$-action through $e$. Each leaf of $\mathfrak{F}_{\mathfrak{b}, \bar{l}_{1}, \cdots, \bar{l}_{\mathrm{k}}}$ meets $\Sigma$ at the only one point. That is, $\Sigma$ is regarded as the leaf space of this foliation. For $\xi_{0} \in \mathfrak{b}$ and $t_{i} \in \mathbb{R}(i=1, \cdots, k)$, we set $x_{\xi_{0}, t_{1}, \cdots, t_{k}}:=\operatorname{Exp} \xi_{0} \cdot \gamma_{\xi^{1}\left(t_{1}\right)} \cdots \cdot \gamma_{\xi^{k}\left(t_{k}\right)}$. Also, denote by $\frac{D}{d s}(\bullet)$ the covariant derivative of vector fields $(\bullet)$ along curves in AN (with respect to the left-invariant metric). The following fact is well-known about the geodesics in rank one symmetric spaces of non-compact type but we shall give the proof.

Lemma 4.1. The velocity vector $\gamma_{\xi^{i}}^{\prime}(\mathrm{s})(\mathrm{i}=1, \cdots, \mathrm{k})$ is described as

$$
\gamma_{\xi^{i}}^{\prime}(s)=\frac{1}{\cosh \left(\left\|\lambda_{i}\right\| s\right)}\left(\widetilde{\xi^{i}}\right)_{\gamma_{\xi^{i}}(s)}-\frac{\tanh \left(\left\|\lambda_{i}\right\| s\right)}{\left\|\lambda_{i}\right\|}\left(\widetilde{\mathrm{H}_{\lambda_{i}}}\right)_{\gamma_{\xi^{i}}(s)}
$$

and $\gamma_{\xi_{0}}^{\prime}(\mathrm{s})$ is described as

$$
\gamma_{\xi_{0}}^{\prime}(s)=\left(\widetilde{\xi}_{0}\right)_{\gamma_{\xi_{0}}(s)}
$$

Proof. Set $Y(s):=\frac{1}{\cosh \left(\left\|\lambda_{i}\right\| s\right)}\left(\widetilde{\xi^{i}}\right)_{\gamma_{\xi^{i}}(s)}-\frac{\tanh \left(\left\|\lambda_{i}\right\| s\right)}{\left\|\lambda_{i}\right\|}\left(\widetilde{\mathrm{H}_{\lambda_{i}}}\right)_{\gamma_{\xi^{i}}(s)}$. It is clear that $Y(0)=\xi^{i}$. By using Lemma 3.4, we can show $\frac{\mathrm{D}}{\mathrm{ds}} Y=0$. Hence we obtain $Y(s)=\gamma_{\xi^{i}}^{\prime}(\mathrm{s})$. Also, it is clear that $\left(\widetilde{\xi}_{0}\right)_{\gamma \xi_{0}(0)}=\xi_{0}$. By using Lemma 3.4, we can show $\frac{\mathrm{D}}{\mathrm{ds}}\left(\widetilde{\xi}_{0}\right)_{\gamma_{\xi_{0}}(s)}=0$. Hence we obtain $\left(\widetilde{\xi}_{0}\right)_{\gamma_{\xi_{0}}(s)}=\gamma_{\xi_{0}}^{\prime}(s)$. q.e.d.

Next we shall show the following fact.

Lemma 4.2. The point $\chi_{\xi_{0}, t_{1}, \cdots, t_{k}}$ belongs to $\Sigma$.

Proof. It is clear that $\operatorname{Exp}\left(\xi_{0}\right)$ belongs to $\Sigma$. First we shall show that $\operatorname{Exp}\left(\xi_{0}\right) \cdot \gamma_{\xi^{1}\left(t_{1}\right)}$ belongs to $\Sigma$. Let $\gamma_{\xi_{0}}$ be the geodesic in $A N$ with $\gamma_{\xi_{0}}^{\prime}(0)=\xi_{0}$. Since $\gamma_{\xi 1}$ is a geodesic in $A N$ and $\mathrm{L}_{\operatorname{Exp}\left(\xi_{0}\right)}$ is an isometry of $A N, \mathrm{~L}_{\operatorname{Exp}\left(\xi_{0}\right)} \circ \gamma_{\xi 1}$ is a geodesic in $A N$. Hence we suffice to show that $\left(\mathrm{L}_{\operatorname{Exp}\left(\xi_{0}\right)} \circ \gamma_{\xi^{1}}\right)^{\prime}(0)=\left(\widetilde{\xi}^{1}\right)_{\operatorname{Exp}\left(\xi_{0}\right)}$ is tangent to $\Sigma$. Denote by $\hat{\xi}^{1}$ the parallel vector field along $\gamma_{\xi_{0}}$. Take orthonormal bases $\left\{e_{1}^{\lambda}, \cdots, e_{m_{\lambda}}^{\lambda}\right\}$ of $\mathfrak{g}_{\lambda}\left(\lambda \in \triangle_{+}\right)$. Also, take an orthonormal base $\left\{e_{1}^{0}, \cdots, e_{r}^{0}\right\}$ 
of a. We describe $\widehat{\xi}^{1}$ as

$$
\left.\widehat{\xi}^{1}(s)=\sum_{i=1}^{r} a_{i}^{0}(s)\left(\widetilde{e_{i}^{0}}\right)_{\gamma_{\xi_{0}}(s)}+\sum_{\lambda \in \triangle_{+}} \sum_{i=1}^{m_{\lambda}} a_{i}^{\lambda}(s) \widetilde{\left(e_{i}^{\lambda}\right.}\right)_{\gamma_{\xi_{0}}(s)} \quad(s \in \mathbb{R}),
$$

where $a_{i}^{0}$ and $a_{i}^{\lambda}$ are functions over $\mathbb{R}$. Fix $s_{0} \in \mathbb{R}$. By using Lemma 3.4, we can show

$$
\begin{aligned}
& \left.\frac{\mathrm{D}}{\mathrm{d} s}\right|_{s=s_{0}} \widehat{\xi}^{1}=\sum_{i=1}^{r}\left(\left(a_{i}^{0}\right)^{\prime}\left(s_{0}\right)\left(\widetilde{e_{i}^{0}}\right)_{\gamma_{\xi_{0}}\left(s_{0}\right)}+\left.\left(a_{i}^{0}\right)\left(s_{0}\right) \frac{D}{d s}\right|_{s=s_{0}}\left(\left(\widetilde{e_{i}^{0}}\right)_{\gamma_{\varepsilon_{0}}(s)}\right)\right) \\
& +\sum_{\lambda \in \Delta_{+}} \sum_{i=1}^{m_{\lambda}}\left(\left(a_{i}^{\lambda}\right)^{\prime}\left(s_{0}\right)\left(\widetilde{e_{i}^{\lambda}}\right)_{\gamma \xi_{0}\left(s_{0}\right)}+\left.a_{i}^{\lambda}\left(s_{0}\right) \frac{D}{d s}\right|_{s=s_{0}}\left(\left(\widetilde{e_{i}^{\lambda}}\right)_{\gamma \xi_{0}(s)}\right)\right) \\
& =\sum_{i=1}^{r}\left(\left(a_{i}^{0}\right)^{\prime}\left(s_{0}\right)\left(\widetilde{e_{i}^{0}}\right)_{\gamma_{\xi_{0}}\left(s_{0}\right)}+\left(a_{i}^{0}\right)\left(s_{0}\right) \nabla_{\gamma_{\varepsilon_{0}}^{\prime}\left(s_{0}\right)}\left(\left(\widetilde{e_{i}^{0}}\right)_{\gamma_{\varepsilon_{0}}\left(s_{0}\right)}\right)\right) \\
& +\sum_{\lambda \in \Delta_{+}} \sum_{i=1}^{m_{\lambda}}\left(\left(a_{i}^{\lambda}\right)^{\prime}\left(s_{0}\right)\left(\widetilde{e_{i}^{\lambda}}\right)_{\gamma_{\varepsilon_{0}}\left(s_{0}\right)}+a_{i}^{\lambda}\left(s_{0}\right) \nabla_{\gamma_{\varepsilon_{0}}^{\prime}\left(s_{0}\right)}\left(\left(\widetilde{e_{i}^{\lambda}}\right)_{\gamma_{\varepsilon_{0}}\left(s_{0}\right)}\right)\right) \\
& =\sum_{i=1}^{r}\left(\left(a_{i}^{0}\right)^{\prime}\left(s_{0}\right)\left(\widetilde{e_{i}^{0}}\right)_{\gamma_{\xi_{0}}\left(s_{0}\right)}+\left(a_{i}^{0}\right)\left(s_{0}\right)\left(\nabla_{\widetilde{\varepsilon_{0}}} \widetilde{e_{i}^{0}}\right)_{\gamma_{\xi_{0}}\left(s_{0}\right)}\right) \\
& \left.+\sum_{\lambda \in \triangle_{+}} \sum_{i=1}^{m_{\lambda}}\left(\left(a_{i}^{\lambda}\right)^{\prime}\left(s_{0}\right)\left(\widetilde{e_{i}^{\lambda}}\right)_{\gamma_{\varepsilon_{0}}\left(s_{0}\right)}+a_{i}^{\lambda}\left(s_{0}\right)\left(\nabla_{\widetilde{\xi_{0}}} \widetilde{e_{i}^{\lambda}}\right)_{\gamma_{\varepsilon_{0}}\left(s_{0}\right)}\right)\right) \\
& =\sum_{i=1}^{r}\left(a_{i}^{0}\right)^{\prime}\left(s_{0}\right)\left(\widetilde{e_{i}^{0}}\right)_{\gamma_{\varepsilon_{0}}\left(s_{0}\right)}+\sum_{\lambda \in \Delta_{+}} \sum_{i=1}^{m_{\lambda}}\left(a_{i}^{\lambda}\right)^{\prime}\left(s_{0}\right)\left(\widetilde{e_{i}^{\lambda}}\right)_{\gamma_{\xi_{0}}\left(s_{0}\right)}=0
\end{aligned}
$$

that is, $\left(a_{i}^{0}\right)^{\prime}\left(s_{0}\right)=\left(a_{i}^{\lambda}\right)^{\prime}\left(s_{0}\right)=0$, where we use $\gamma_{\xi_{0}}^{\prime}\left(s_{0}\right)=\widetilde{\xi}_{0 \gamma_{\xi_{0}}}\left(s_{0}\right)$. From the arbitrariness of $s_{0}$, we see that $a_{i}^{0}$ and $a_{i}^{\lambda}$ are constant. Hence we obtain $\widehat{\xi}^{1}(s)=\left(\widetilde{\xi}^{1}\right)_{\gamma_{\varepsilon_{0}}(s)}$. On the other hand, since $\xi^{1}$ is tangent to $\Sigma$ and $\Sigma$ is totally geodesic, $\hat{\xi}^{1}(1)$ also is tangent to $\Sigma$. Hence we see that $\left(\widetilde{\xi}^{1}\right)_{\operatorname{Exp}\left(\xi_{0}\right)}$ is tangent to $\Sigma$. Therefore $\operatorname{Exp}\left(\xi_{0}\right) \cdot \gamma_{\xi^{1}\left(t_{1}\right)}$ belongs to $\Sigma$.

Next we shall show that $\operatorname{Exp}\left(\xi_{0}\right) \cdot \gamma_{\xi^{1}\left(t_{1}\right)} \cdot \gamma_{\xi^{2}\left(t_{2}\right)}$ belongs to $\Sigma$. Since $\gamma_{\xi^{2}}$ is a geodesic in $A N$ and $L_{\operatorname{Exp}\left(\xi_{0}\right) \cdot \gamma_{\xi_{1}}\left(t_{1}\right)}$ is an isometry of $A N, L_{\operatorname{Exp}\left(\xi_{0}\right) \cdot \gamma_{\xi_{1}}\left(t_{1}\right)} \circ \gamma_{\xi^{2}}$ is a geodesic in $A N$. Hence we suffice to show that $\left(\mathrm{L}_{\operatorname{Exp}\left(\xi_{0}\right) \cdot \gamma_{\xi_{1}}\left(\mathrm{t}_{1}\right)} \circ \gamma_{\xi^{2}}\right)^{\prime}(0)=\left(\widetilde{\xi}^{2}\right)_{\operatorname{Exp}\left(\xi_{0}\right) \cdot \gamma_{\xi_{1}}\left(\mathrm{t}_{1}\right)}$ is tangent to $\Sigma$. Denote by $\widehat{\xi}^{2}$ the parallel vector field along $\bar{\gamma}_{\xi_{1}}:=\mathrm{L}_{\operatorname{Exp}\left(\xi_{0}\right)} \circ \gamma_{\xi^{1}}$ with $\widehat{\xi}^{2}(0)=\left(\widetilde{\xi}^{2}\right)_{\operatorname{Exp}\left(\xi_{0}\right)}$. We describe $\widehat{\xi}^{2}$ as

$$
\widehat{\xi}^{2}(s)=\sum_{i=1}^{r} b_{i}^{0}(s)\left(\widetilde{e_{i}^{0}}\right)_{\bar{\gamma}_{\xi 1}(s)}+\sum_{\lambda \in \triangle_{+}} \sum_{i=1}^{m_{\lambda}} b_{i}^{\lambda}(s)\left(\widetilde{e_{i}^{\lambda}}\right)_{\bar{\gamma}_{\xi 1}(s)} \quad(s \in \mathbb{R}),
$$


where $b_{i}^{o}$ and $b_{i}^{\lambda}$ are functions over $\mathbb{R}$. Fix $s_{0} \in \mathbb{R}$. By using Lemma 3.4, we can show

$$
\begin{aligned}
& \left.\frac{\mathrm{D}}{\mathrm{d} s}\right|_{s=s_{0}} \widehat{\xi}^{2}=\sum_{i=1}^{\mathrm{r}}\left(\left(b_{i}^{0}\right)^{\prime}\left(s_{0}\right)\left(\widetilde{e_{i}^{0}}\right)_{\bar{\gamma}_{\xi, 1}\left(s_{0}\right)}+\left.\left(b_{i}^{0}\right)\left(s_{0}\right) \frac{\mathrm{D}}{\mathrm{ds}}\right|_{s=s_{0}}\left(\left(\widetilde{e_{i}^{\mathcal{O}}}\right)_{\bar{\gamma}_{\xi},(s)}\right)\right) \\
& +\sum_{\lambda \in \Delta_{+}} \sum_{i=1}^{m_{\lambda}}\left(\left(b_{i}^{\lambda}\right)^{\prime}\left(s_{0}\right)\left(\widetilde{e_{i}^{\lambda}}\right)_{\bar{\gamma}_{\xi 1}\left(s_{0}\right)}+\left.b_{i}^{\lambda}\left(s_{0}\right) \frac{D}{d s}\right|_{s=s_{0}}\left(\left(\widetilde{e_{i}^{\lambda}}\right)_{\bar{\gamma}_{\xi 1}(s)}\right)\right) \\
& =\sum_{i=1}^{r}\left(\left(b_{i}^{0}\right)^{\prime}\left(s_{0}\right)\left(\widetilde{e_{i}^{0}}\right)_{\bar{\gamma}_{\xi 1}\left(s_{0}\right)}+\left(b_{i}^{0}\right)\left(s_{0}\right) \nabla_{\bar{\gamma}_{\xi 1}^{\prime}\left(s_{0}\right)}\left(\left(\widetilde{e_{i}^{0}}\right)_{\bar{\gamma}_{\xi 1}(s)}\right)\right) \\
& \left.\left.+\sum_{\lambda \in \triangle_{+}} \sum_{i=1}^{m_{\lambda}}\left(\left(b_{i}^{\lambda}\right)^{\prime}\left(s_{0}\right) \widetilde{\left(e_{i}^{\lambda}\right.}\right)_{\bar{\gamma}_{\xi 1}\left(s_{0}\right)}+b_{i}^{\lambda}\left(s_{0}\right) \nabla_{\bar{\gamma}_{\xi 1}^{\prime}\left(s_{0}\right)}\left(\widetilde{e_{i}^{\lambda}}\right)_{\bar{\gamma}_{\xi 1}(s)}\right)\right)=0 .
\end{aligned}
$$

Since $\gamma_{\xi^{1}}^{\prime}\left(s_{0}\right)=\frac{1}{\cosh \left(\left\|\lambda_{1}\right\| s_{0}\right)}(\widetilde{\xi})_{\gamma_{\xi^{1}}\left(s_{0}\right)}-\frac{\tanh \left(\left\|\lambda_{1}\right\| s_{0}\right)}{\left\|\lambda_{1}\right\|}\left(\widetilde{\mathrm{H}_{\lambda_{1}}}\right)_{\gamma_{\xi^{1}}\left(s_{0}\right)}$ by Lemma 4.1, $\bar{\gamma}_{\xi^{1}}^{\prime}\left(s_{0}\right)$ is described as

$$
\begin{aligned}
& \bar{\gamma}_{\xi^{1}}^{\prime}\left(s_{0}\right)=\left(\mathrm{L}_{\operatorname{Exp}\left(\xi_{0}\right)}\right)_{*}\left(\gamma_{\xi^{1}}^{\prime}\left(s_{0}\right)\right) \\
& =\frac{1}{\cosh \left(\left\|\lambda_{1}\right\| s_{0}\right)}\left(\widetilde{\xi^{1}}\right)_{\bar{\gamma}_{\xi^{1}}\left(s_{0}\right)}-\frac{\tanh \left(\left\|\lambda_{1}\right\| s_{0}\right)}{\left\|\lambda_{1}\right\|}\left(\widetilde{\mathrm{H}}_{\lambda_{1}}\right)_{\bar{\gamma}_{\xi^{1}}\left(s_{0}\right)} .
\end{aligned}
$$

Hence, by using Lemma 3.4, we have

$$
\begin{aligned}
\nabla_{\bar{\gamma}_{\xi^{1}}^{\prime}\left(s_{0}\right)}\left(\left(\widetilde{e_{i}^{0}}\right)_{\bar{\gamma}_{\xi_{1}}}\right. & =\frac{1}{\cosh \left(\left\|\lambda_{1}\right\| s_{0}\right)}\left(\nabla_{\widetilde{\xi}^{1}} \widetilde{e_{i}^{0}}\right)_{\bar{\gamma}_{\xi^{1}}\left(s_{0}\right)}-\frac{\tanh \left(\left\|\lambda_{1}\right\| s_{0}\right)}{\left\|\lambda_{1}\right\|}\left(\nabla_{\widetilde{H_{\lambda_{1}}}} \widetilde{e_{i}^{0}}\right)_{\bar{\gamma}_{\xi^{1}}\left(s_{0}\right)} \\
& =-\frac{\lambda_{1}\left(e_{i}^{0}\right)}{\cosh \left(\left\|\lambda_{1}\right\| s_{0}\right)}\left(\widetilde{\xi^{1}}\right)_{\bar{\gamma}_{\xi^{1}}\left(s_{0}\right)}
\end{aligned}
$$

and

$$
\begin{aligned}
\nabla_{\bar{\gamma}_{\xi 1}^{\prime}\left(s_{0}\right)}\left(\widetilde{e_{i}^{\lambda}}\right)_{\bar{\gamma}_{\xi 1}}=\frac{1}{\cosh \left(\left\|\lambda_{1}\right\| s_{0}\right)}\left(\nabla_{\widetilde{\xi^{1}}} \widetilde{e_{i}^{\lambda}}\right)_{\bar{\gamma}_{\xi^{1}}\left(s_{0}\right)}-\frac{\tanh \left(\left\|\lambda_{1}\right\| s_{0}\right)}{\left\|\lambda_{1}\right\|}\left(\nabla_{\left.\widetilde{H_{\lambda_{1}}} \widetilde{e_{i}^{\lambda}}\right)_{\bar{\gamma}_{\xi^{1}}\left(s_{0}\right)}}\right. \\
= \begin{cases}\left.\left.\frac{1}{2 \cosh \left(\left\|\lambda_{1}\right\| s_{0}\right)}\left(\widetilde{\xi^{1}}, \widetilde{e_{i}^{\lambda}}\right]+\widetilde{\theta\left[e_{i}^{\lambda}, \theta \xi^{1}\right.}\right]\right) & \left(\lambda_{1}-\lambda \in \triangle_{+}\right) \\
\frac{1}{2 \cosh \left(\left\|\lambda_{1}\right\| s_{0}\right)}\left(\left[\widetilde{\xi}, \widetilde{e_{i}^{\lambda}}\right]+2\left\langle\widetilde{\xi^{1}}, \widetilde{e_{i}^{\lambda}}\right\rangle \widetilde{H}_{\lambda_{1}}\right) & \left(\lambda_{1}=\lambda\right) \\
\frac{1}{2 \cosh \left(\left\|\lambda_{1}\right\| s_{0}\right)}\left(\widetilde{\xi^{1}}, \widetilde{e_{i}^{\lambda}}\right]+\widetilde{\left.\theta\left[\xi^{1}, \theta e_{i}^{\lambda}\right]\right)} & \left(\lambda-\lambda_{1} \in \triangle_{+}\right) \\
\frac{1}{2 \cosh \left(\left\|\lambda_{1}\right\| s_{0}\right)}\left[\widetilde{\xi^{1}}, \widetilde{e_{i}^{\lambda}}\right] & \left(\lambda_{1}-\lambda \notin \Delta \cup\{0\}\right) .\end{cases}
\end{aligned}
$$


By substituting (4.4) and (4.5) into (4.3), we obtain

$$
\begin{aligned}
& \left.\frac{\mathrm{D}}{\mathrm{d} s}\right|_{s=s_{0}} \widehat{\xi}^{2}=\sum_{i=1}^{\mathrm{r}}\left(\left(b_{i}^{\mathcal{O}}\right)^{\prime}\left(s_{0}\right)\left(\widetilde{e_{i}^{\mathcal{O}}}\right)_{\bar{\gamma}_{\xi, 1}\left(s_{0}\right)}-\frac{\lambda_{1}\left(e_{i}^{\mathcal{O}}\right)\left(b_{i}^{\mathcal{O}}\right)\left(s_{0}\right)}{\cosh \left(\left\|\lambda_{1}\right\| s_{0}\right)}\left(\widetilde{\xi^{1}}\right)_{\bar{\gamma}_{\xi, 1}\left(s_{0}\right)}\right) \\
& +\sum_{\lambda \in \Delta_{+}} \sum_{i=1}^{m_{\lambda}}\left(b_{i}^{\lambda}\right)^{\prime}\left(s_{0}\right)\left(\widetilde{e_{i}^{\lambda}}\right)_{\bar{\gamma}_{\xi 1}\left(s_{0}\right)} \\
& \left.\left.+\sum_{\lambda_{1}-\lambda \in \Delta_{+}} \sum_{i=1}^{m_{\lambda}} \frac{b_{i}^{\lambda}\left(s_{0}\right)}{2 \cosh \left(\left\|\lambda_{1}\right\| s_{0}\right)}\left(\widetilde{[\xi 1}, \widetilde{e_{i}^{\lambda}}\right]+\widetilde{\theta\left[e_{i}^{\lambda}, \theta\right.} \xi^{1}\right]\right) \\
& \left.+\sum_{\lambda-\lambda_{1} \in \Delta_{+}} \sum_{i=1}^{m_{\lambda}} \frac{b_{i}^{\lambda}\left(s_{0}\right)}{2 \cosh \left(\left\|\lambda_{1}\right\| s_{0}\right)}\left(\widetilde{\left.\xi^{1}, \widetilde{e_{i}^{\lambda}}\right]}+\widetilde{\theta\left[\xi^{1}, \theta\right.} e_{i}^{\lambda}\right]\right) \\
& +\sum_{\lambda-\lambda_{1} \notin \Delta \cup\{0\}} \sum_{i=1}^{m_{\lambda}} \frac{b_{i}^{\lambda}\left(s_{0}\right)}{2 \cosh \left(\left\|\lambda_{1}\right\| s_{0}\right)}\left[\widetilde{\xi 1}, \widetilde{e_{i}^{\lambda}}\right] \\
& +\sum_{i=1}^{m_{\lambda_{1}}} \frac{b_{i}^{\lambda_{1}}\left(s_{0}\right)}{2 \cosh \left(\left\|\lambda_{1}\right\| s_{0}\right)}\left(\left[\widetilde{\xi}^{1}, \widetilde{e_{i}^{\lambda_{1}}}\right]+2\left\langle\widetilde{\xi^{1}}, \widetilde{e_{i}^{\lambda_{1}}}\right\rangle \widetilde{H}_{\lambda_{1}}\right)=0 .
\end{aligned}
$$

Without loss of generality, we may assume that $e_{1}^{\lambda_{2}}=\xi^{2}$. Hence we have $b_{1}^{\lambda_{2}}(0)=1$ and $b_{i}^{\lambda}(0)=0$ for any $(\lambda, i)$ other than $\left(\lambda_{2}, 1\right)$. From (4.6) and these relations, we obtain $b_{1}^{\lambda_{2}} \equiv 1$ and $b_{i}^{\lambda} \equiv 0$ for any $(\lambda, i)$ other than $\left(\lambda_{2}, 1\right)$, where we note that $\lambda_{1}-\lambda_{2} \notin \Delta \cup\{0\}$. Therefore we obtain $\widehat{\xi^{2}}=\left(\widetilde{\xi^{2}}\right)_{\bar{\gamma}_{\xi_{1}}(s)}$. On the other hand, since $\left(\widehat{\xi^{2}}\right)(0)$ is tangent to $\Sigma$ and $\Sigma$ is totally geodesic, $\widehat{\xi}^{2}\left(t_{1}\right)$ also is tangent to $\Sigma$. Hence we see that $\left(\widetilde{\xi^{2}}\right)_{\operatorname{Exp}\left(\xi_{0}\right) \cdot \gamma_{\xi_{1}}\left(t_{1}\right)}$ is tangent to $\Sigma$. Therefore $\operatorname{Exp}\left(\xi_{0}\right)$. $\gamma_{\xi^{1}\left(t_{1}\right)} \cdot \gamma_{\xi^{2}\left(t_{2}\right)}$ belongs to $\Sigma$. In the sequel, by repeating the same discussion, we can derive that $x_{\xi^{0}, t_{1}, \cdots, t_{k}}=\operatorname{Exp}\left(\xi_{0}\right) \cdot \gamma_{\xi^{1}\left(t_{1}\right) \cdots \cdots} \gamma_{\xi^{k}\left(t_{k}\right)}$ belongs to $\Sigma$.

It is clear that any point of $\Sigma$ is described as $x_{\xi_{0}, t_{1}, \cdots, t_{k}}$ for some $\xi_{0} \in \mathfrak{b}$ and some $t_{1}, \cdots, t_{k} \in$ $\mathbb{R}$. Fix an orthonormal base $\left\{e_{1}^{0}, \cdots, e_{\mathfrak{m}_{0}}^{0}\right\}$ of $\mathfrak{b}$, where $\mathfrak{m}_{0}:=\operatorname{dim} \mathfrak{b}$. Define vector fields $E_{i}^{0}$ $\left(i=1, \cdots, m_{0}\right)$ and $E^{j}(j=1, \cdots, k)$ along $\Sigma$ by

$$
\begin{aligned}
& \left(E_{i}^{0}\right)_{x_{\varepsilon_{0}, t_{1}}, \cdots, t_{k}}:=\left(L_{x_{\varepsilon_{0}, t_{1}}, \ldots, t_{k}}\right)_{*}\left(e_{i}^{0}\right)\left(=\left(\widetilde{\tilde{e_{i}^{0}}}\right)_{x_{\varepsilon_{0}, t_{1}}, \cdots, t_{k}}\right) \\
& \text { and } \quad\left(E^{j}\right)_{x_{\xi_{0}, t_{1}, \cdots, t_{k}}}:=\left(L_{x_{\varepsilon_{0}, t_{1}, \cdots, t_{k}}}\right)_{*}\left(\xi_{t_{j}}^{j}\right)\left(=\left(\widetilde{\xi_{t_{j}}^{j}}\right)_{x_{\xi_{0}, t_{1}, \cdots, t_{k}}}\right) \text {. }
\end{aligned}
$$

By imitating the discussions in the proofs of Lemmas 4.1 and 4.2 , we can show the following fact for these vector fields.

Lemma 4.3. The vector fields $\mathrm{E}_{\mathfrak{i}}^{0}\left(i=1, \cdots, \mathrm{m}_{0}\right)$ and $\mathrm{E}^{j}(j=1, \cdots, k)$ are tangent to $\Sigma$ and they give a parallel orthonormal tangent frame field on $\Sigma$.

Proof. Let $\left(\widehat{\xi}^{i}\right)^{j}\left(\right.$ resp. $\left.\left(\widehat{\xi}^{i}\right)^{0}\right)$ be the parallel vector field along $\gamma_{\xi^{j}}(\mathfrak{i} \neq j)\left(\right.$ resp. $\left.\gamma_{\xi_{0}}\right)$ with $\left(\widehat{\xi}^{i}\right)_{0}^{j}=$ $\xi^{i}$ (resp. $\left.\left(\widehat{\xi^{i}}\right)_{0}^{0}=\xi^{i}\right)$ and $\left(\widehat{\xi_{0}}\right)^{j}$ be the parallel vector field along $\gamma_{\xi^{j}}$ with $\left(\widehat{\xi_{0}}\right)_{0}^{j}=\xi_{0}$. According to Lemma 4.1, we have $\left(\gamma_{\xi^{i}}\right)^{\prime}(\mathrm{t})=\left(\mathrm{L}_{\gamma_{\xi^{i}}(\mathrm{t})}\right)_{*}\left(\xi_{\mathrm{t}}^{\mathrm{i}}\right)$ and $\left(\gamma_{\xi_{0}}\right)^{\prime}(\mathrm{t})=\left(\mathrm{L}_{\gamma_{\xi_{0}}}(\mathrm{t})\right)_{*}\left(\xi_{0}\right)$. Also, we can 
$\operatorname{show}\left(\widehat{\xi^{i}}\right)_{\gamma_{\xi j}(t)}^{j}=\left(L_{\gamma_{\xi j}(t)}\right)_{*}\left(\xi^{i}\right)(j \neq i),\left(\widehat{\xi^{i}}\right)_{\gamma_{\xi_{0}}(t)}^{0}=\left(L_{\gamma_{\xi_{0}}(t)}\right)_{*}\left(\xi^{i}\right)$ and $\left(\widehat{\xi_{0}}\right)_{\gamma_{\xi j}(t)}^{j}=\left(L_{\gamma_{\xi^{j}}}(t)\right)_{*}\left(\xi_{0}\right)$ by imitating the discussion in the proof of Lemma 4.2. On the basis of these facts, we can derive the statement of this lemma, where we note that $\Sigma$ is flat.

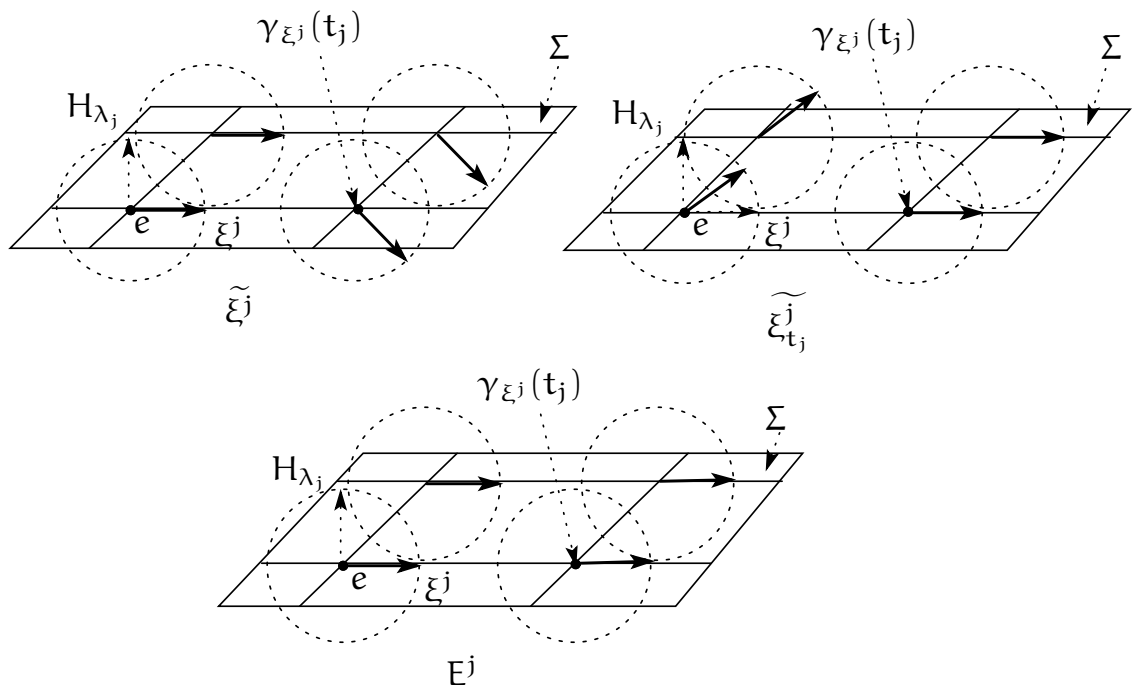

Figure 4 .

By using these lemmas, we prove Theorem A.

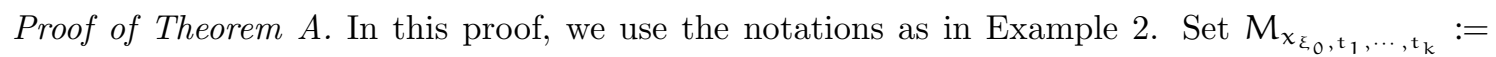
$\mathrm{S}_{\mathfrak{b}, \bar{l}_{1}, \cdots, \bar{l}_{k}} \cdot x_{\xi_{0}, t_{1}, \cdots, t_{k}}$. Denote by $H^{x_{\xi_{0}, t_{1}, \cdots, t_{k}}}$ the mean curvature vector field of $M_{x_{\xi_{0}, t_{1}, \cdots, t_{k}}}$. Let $\left\{e_{1}^{0}, \cdots, e_{m_{0}}^{0}\right\}$ be an orthonormal base of $\mathfrak{b}$ and $\left(H_{\lambda}\right)_{\mathfrak{b}}=\sum_{i=1}^{m_{0}} H_{\lambda}^{i} e_{i}^{0}$ be the $\mathfrak{b}$-component of $\mathrm{H}_{\lambda}$. According to the fact (iv) stated in Introduction, we have

$$
M_{x_{\xi_{0}, t_{1}, \cdots, t_{k}}}=L_{x_{\xi_{0}, t_{1}, \cdots, t_{k}}}\left(S_{\mathfrak{b}, l_{\xi_{1}, t_{1}}, \cdots, l_{\xi_{k}, t_{k}}} \cdot e\right) .
$$

Denote by $\widehat{H}^{\xi_{0}}, t_{1}, \cdots, t_{k}$ the mean curvature vector field of $S_{\mathfrak{b}, l_{\xi^{1}, t_{1}}, \cdots, l_{\xi^{k}, t_{k}}} \cdot e$. According to Lemma 3.1 , we have

$$
\left(\widehat{H}^{\xi_{0}, t_{1}, \cdots, t_{k}}\right)_{e}=\sum_{\lambda \in \triangle_{+}} m_{\lambda}\left(H_{\lambda}\right)_{\mathfrak{b}}-\sum_{i=1}^{k}\left\|\lambda_{i}\right\| \tanh \left(\left\|\lambda_{i}\right\| t_{i}\right)\left(m_{\lambda_{i}}+2 m_{2 \lambda_{i}}\right) \xi_{t_{i}}^{i}
$$

and hence

$$
\begin{aligned}
& \left(H^{x_{\xi_{0}, t_{1}, \cdots, t_{k}}}\right)_{x_{\xi_{0}, t_{1}, \cdots, t_{k}}}=\sum_{\lambda \in \triangle_{+}} \sum_{i=1}^{m_{0}} m_{\lambda} H_{\lambda}^{i}\left(E_{i}^{0}\right)_{x_{\xi_{0}, t_{1}, \cdots, t_{k}}} \\
& -\sum_{i=1}^{k}\left\|\lambda_{i}\right\| \tanh \left(\left\|\lambda_{i}\right\| t_{i}\right)\left(m_{\lambda_{i}}+2 m_{2 \lambda_{i}}\right)\left(E^{i}\right)_{\chi_{\xi_{0}, t_{1}, \cdots, t_{k}}} .
\end{aligned}
$$


Define a tangent vector field $Z$ over $\Sigma$ by $Z_{x}:=\left(H^{x}\right)_{x}(x \in \Sigma)$. According to (4.7), we have

$$
\begin{aligned}
\mathrm{Z}_{x_{\varepsilon_{0}, t_{1}, \cdots, t_{k}}=} & \sum_{\lambda \in \triangle_{+}} \sum_{i=1}^{m_{0}} m_{\lambda} H_{\lambda}^{i}\left(E_{i}^{0}\right)_{x_{\varepsilon_{0}, t_{1}}, \ldots, t_{k}} \\
& -\sum_{i=1}^{k}\left\|\lambda_{i}\right\| \tanh \left(\left\|\lambda_{i}\right\| t_{i}\right)\left(m_{\lambda_{i}}+2 m_{2 \lambda_{i}}\right)\left(E^{i}\right)_{x_{\varepsilon_{0}, t_{1}}, \cdots, t_{k}} .
\end{aligned}
$$

Define a coordinate $\phi=\left(\mathrm{u}_{1}, \cdots, \mathrm{u}_{\mathrm{m}_{\mathrm{o}}+\mathrm{k}}\right): \Sigma \rightarrow \mathbb{R}^{\mathrm{m}_{\mathrm{o}}+\mathrm{k}}$ of $\Sigma$ by

$$
\phi\left(x_{\sum_{i=1}^{m_{0}} s_{i} e_{i}^{o}, t_{1}, \cdots, t_{k}}\right):=\left(s_{1}, \cdots, s_{m_{0}}, t_{1}, \cdots, t_{k}\right)
$$

$\left(s_{1}, \cdots, s_{m_{0}}, t_{1}, \cdots, t_{k} \in \mathbb{R}\right)$. We can show $\frac{\partial}{\partial u_{i}}=E_{i}^{0}\left(i=1, \cdots, m_{0}\right)$ and $\frac{\partial}{\partial u_{m_{0}+j}}=E^{j}(j=$ $1, \cdots, k)$. Hence $\phi$ is a Euclidean coordinate of $\Sigma$. Under the identification of $\Sigma$ and $\mathbb{R}^{m_{0}+k}$ by $\phi$, we regard $Z$ as a tangent vector field on $\mathbb{R}^{m_{0}+k}$. Then $Z$ is described as

$$
\begin{aligned}
\mathrm{Z}_{\left(\mathrm{u}_{1}, \cdots, u_{\mathrm{m}_{\mathrm{o}}+\mathrm{k}}\right)=} & \sum_{\lambda \in \triangle_{+}} \mathrm{m}_{\lambda} \mathrm{H}_{\lambda}^{1}, \cdots, \sum_{\lambda \in \triangle_{+}} \mathrm{m}_{\lambda} \mathrm{H}_{\lambda}^{\mathrm{m}_{\mathrm{o}}}, \\
& -\left\|\lambda_{1}\right\| \tanh \left(\left\|\lambda_{1}\right\| \mathrm{u}_{\mathrm{m}_{\mathrm{o}}+1}\right)\left(\mathrm{m}_{\lambda_{1}}+2 \mathrm{~m}_{2 \lambda_{1}}\right), \\
& \left.\cdots,-\left\|\lambda_{k}\right\| \tanh \left(\left\|\lambda_{k}\right\| \mathrm{u}_{\mathrm{m}_{\mathrm{o}}+\mathrm{k}}\right)\left(\mathrm{m}_{\lambda_{k}}+2 \mathrm{~m}_{2 \lambda_{k}}\right)\right) .
\end{aligned}
$$

Fix $\left(a_{1}, \cdots, a_{m_{0}}, t_{1}, \cdots, t_{k}\right) \in \mathbb{R}^{m_{0}+k}$. Let $c$ be the integral curve of $Z$ starting from $\left(a_{1}, \cdots, a_{m_{0}}, t_{1}, \cdots, t_{k}\right)$ and let $c=\left(c_{1}, \cdots, c_{m_{0}+k}\right)$. We suffice to investigate $c$ to investigate the mean curvature flow starting from $M_{x_{\sum_{i=1}^{m_{0}} a_{i} e_{i}^{0}, t_{1}, \cdots, t_{k}}}$ From $c^{\prime}(t)=Z_{c(t)}$, we have $c_{i}^{\prime}(t)=\sum_{\lambda \in \Delta_{+}} m_{\lambda} H_{\lambda}^{i}(i=$ $\left.1, \cdots, m_{0}\right)$ and $c_{m_{0}+j}^{\prime}(t)=-\left(m_{\lambda_{j}}+2 m_{2 \lambda_{j}}\right)\left\|\lambda_{j}\right\| \tanh$

$\left(\left\|\lambda_{j}\right\| c_{m_{0}+j}(t)\right)(j=1, \cdots, k)$. By solving $c_{i}^{\prime}(t)=\sum_{\lambda \in \Delta_{+}} m_{\lambda} H_{\lambda}^{i}$ under the initial condition $c_{i}(0)=a_{i}$, we have

$$
c_{i}(t)=a_{i}+t \sum_{\lambda \in \triangle_{+}} m_{\lambda} H_{\lambda}^{i}
$$

Also, by solving $c_{m_{0}+j}^{\prime}(t)=-\left(m_{\lambda_{j}}+2 m_{2 \lambda_{j}}\right)\left\|\lambda_{j}\right\| \tanh \left(\left\|\lambda_{j}\right\| c_{m_{0}+j}(t)\right)$ under the initial condition $c_{m_{0}+j}(0)=t_{j}$, we have

$$
c_{m_{0}+j}(t)=\frac{1}{\left\|\lambda_{j}\right\|} \operatorname{arcsinh}\left(e^{-\left\|\lambda_{j}\right\|^{2}\left(m_{\lambda_{j}}+2 m_{2 \lambda_{j}}\right) t} \sinh \left(\left\|\lambda_{j}\right\| t_{j}\right)\right)
$$

From (4.10) and (4.11), we can derive $T=\infty, \lim _{t \rightarrow \infty} \sum_{i=1}^{m_{0}} c_{i}(t)^{2}=\infty\left(i=1, \cdots, m_{0}\right)$ and $\lim _{t \rightarrow \infty} c_{m_{0}+j}(t)=0(j=1, \cdots, k)$. If $t_{1}=\cdots=t_{k}=0$, then we have $c_{m_{0}+j} \equiv 0\left(j=1, \cdots, m_{0}\right)$. Hence the mean curvature flow starting from $M_{x_{\xi_{0}, 0, \ldots, 0}}\left(x_{\xi_{0}, 0, \cdots, 0} \in \operatorname{Exp}(\mathfrak{b})\right)$ consists of the leaves of $\mathfrak{F}_{\mathfrak{b}, \bar{l}_{1}, \ldots, \bar{l}_{k}}$ through points of $\operatorname{Exp}(\mathfrak{b})$. Also, according to the fact (iv) stated in Introduction, the leaves of $\mathfrak{F}_{\mathfrak{b}, \bar{l}_{1}, \ldots, \bar{l}_{\mathfrak{k}}}$ through points of $\operatorname{Exp}(\mathfrak{b})$ are congruent to $S_{\mathfrak{b}, \bar{l}_{1}, \cdots, \bar{l}_{k}} \cdot e$. Therefore, the mean curvature flow starting from $M_{x_{\varepsilon_{0}, 0, \ldots, 0}}$ is self-similar. From $\lim _{t \rightarrow \infty} \sum_{i=1}^{m_{0}} c_{i}(t)^{2}=\infty$ $\left(i=1, \cdots, m_{0}\right)$ and $\lim _{t \rightarrow \infty} c_{m_{0}+j}(t)=0(j=1, \cdots, k)$, we see that the mean curvature flow starting 


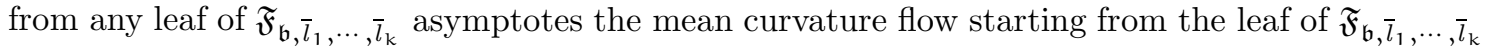
passing through a point of $\operatorname{Exp}(\mathfrak{b})$ q.e.d.

According to this proof, we obtain the following fact.

Corollary 4.1. (i) The mean curvature flow starting from $M_{x_{\varepsilon_{0}, 0, \ldots, 0}}$ is self-similar.

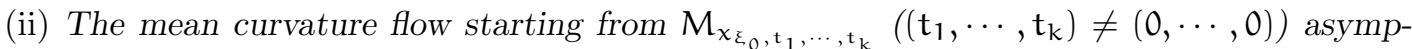
totes the flow starting from $M_{x_{\varepsilon_{0}, 0, \ldots, 0}}$. In more detail, the distance between $M_{x_{\varepsilon_{0}, t_{1}, \cdots, t_{k}}}$ and $\mathrm{M}_{\mathrm{x}_{\xi_{0}, 0, \ldots, 0}}$ is equal to

$$
\sqrt{\sum_{j=1}^{k} \frac{1}{\left\|\lambda_{j}\right\|^{2}} \operatorname{arcsinh}^{2}\left(e^{-\left\|\lambda_{j}\right\|^{2}\left(m_{\lambda_{j}}+2 m_{2 \lambda_{j}}\right) t} \sinh \left(\left\|\lambda_{j}\right\| t_{j}\right)\right)}
$$

which converges to zero as $\mathrm{t} \rightarrow \infty$.

Next we prove Theorem B.

Proof of Theorem B. In case of $\mathfrak{b}=\{0\}$, the relation (4.9) is as follows:

$$
\begin{array}{r}
Z_{\left(u_{1}, \cdots, u_{k}\right)}=\left(-\left\|\lambda_{1}\right\| \tanh \left(\left\|\lambda_{1}\right\| u_{m_{0}+1}\right)\left(m_{\lambda_{1}}+2 m_{2 \lambda_{1}}\right),\right. \\
\left.\cdots,-\left\|\lambda_{k}\right\| \tanh \left(\left\|\lambda_{k}\right\| u_{m_{0}+k}\right)\left(m_{\lambda_{k}}+2 m_{2 \lambda_{k}}\right)\right) .
\end{array}
$$

Hence, according to the dicussion in the proof of Theorem A, the mean curvature flow starting

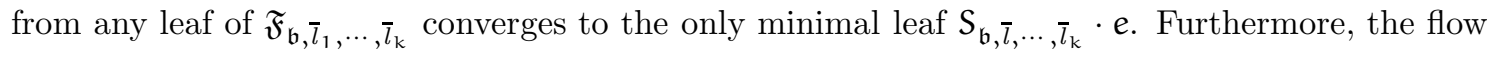
converges to the minimal leaf in $C^{\infty}$-topology because the flow consists of $S_{\mathfrak{b}, \bar{l}_{1}, \cdots, \bar{l}_{k}}$-orbits and the limit submanifold also is a $S_{\mathfrak{b}, \bar{l}_{1}, \ldots, \bar{l}_{k}}$-orbit. q.e.d. 


\section{References}

[1] M. M. Alexandrino and M. Radeschi, Mean curvature flow of singular Riemannian foliations, J. Geom. Anal. 26 2204-2220 (2015).

[2] B. Andrews and C. Baker, Mean curvature flow of pinched submanifolds to spheres, J. Differential Geom. 85 (2010) 357-396.

[3] G. Huisken, Flow by mean curvature of convex surfaces into spheres, J. Differential Geom. 20 (1984) 237-266.

[4] G. Huisken, Contracting convex hypersurfaces in Riemannian manifolds by their mean curvature, Invent. math. 84 (1986) 463-480.

[5] N. Koike, Examples of a complex hyperpolar action without singular orbit, Cubo A Math. J. 12 (2010) 131-147.

[6] N. Koike, Collapse of the mean curvature flow for equifocal submanifolds, Asian J. Math. 15 (2011) 101-128.

[7] N. Koike, Collapse of the mean curvature flow for isoparametric submanifolds in a symmetric space of non-compact type, Kodai Math. J. 37 (2014) 355-382.

[8] J. Milnor, Curvatures of left invariant metrics on Lie groups, Adv. Math. 21 (1976) 293-329. 